\title{
MBER Transmit Precoding for the Rank-Deficient MIMO-Aided Internet of Things
}

\author{
K. Satyanarayana*, Mohammed El-Hajjar*, Ping-Heng $\mathrm{Kuo}^{\dagger}$, Alain Mourad ${ }^{\dagger}$, Lajos Hanzo* \\ ${ }^{*}$ Department of Electronics and Computer Science, University of Southampton, UK. \\ ${ }^{\dagger}$ InterDigital Europe Ltd., London, UK. \\ Email: $\{$ ks1r15, meh, lh\}@ecs.soton.ac.uk, \{ping-heng.kuo, alain.mourad $\} @$ InterDigital.com
}

\begin{abstract}
The Internet of Things (IoT) will support a massive number of devices, which will be connected to the wireless network. In the wireless IoT, the base station serves a wide variety of devices in the same time-frequency resource, where it is expected that the number of devices will be greater than the number of base station antennas. This results in a rank-deficient system. In this paper, we propose a minimum bit error ratio (MBER) precoder for rank-deficient MIMO systems in the context of the IoT, where the IoT devices are generally stationary. We invoke the particle swarm optimization (PSO) algorithm for solving the non-linearly constrained MBER problem and we show that the PSO assisted MBER precoder outperforms the conventional zero forcing and linear minimum mean squared error (LMMSE) precoders, which produce an error floor in these challenging rankdeficient scenarios.
\end{abstract}

\section{INTRODUCTION}

With the ever-growing advances in technology, a wide variety of devices will be connected to the Internet of Things (IoT) [1]. The IoT is expected to be supported by fifth generation (5G) mobile communications. Wireless communication plays a pivotal role in the IoT networking of diverse devices, which will be equipped with microcontrollers and transceivers for communication [2]. However, the enabling wireless IoT poses challenges both in terms of its energy consumption and the paucity of spectral resources to accommodate a large number of devices [1], [3]. The base station can employ diverse multiple-access transmission techniques, such as time division multiple access (TDMA), frequency division multiple access (FDMA) and space division multiple access (SDMA) to serve all the objects simultaneously. In SDMA, the base station exploits the knowledge of the channel response about to be encountered by the distant receivers and pre-processes the signal using a digital transmit precoder (TPC) before transmission to eliminate the interference at the transmitter, so that low-complexity single-user detectors can be invoked by the receivers. To serve a large number of devices within the limited bandwidth, Ding et al. [4] proposed multi-input multi-output non-orthogonal multiple access (MIMO-NOMA) for smallpacket transmissions in the IoT. In this design, the devices having different channel conditions share the same spectral resources, while invoking successive interference cancellation to remove the interference caused by other devices. Furthermore, Tian et al. [5] introduced the concept of overlapping user grouping for increasing the system capacity. In this work, a greedy-search based algorithm is invoked for mitigating the effects of overlap amongst the selected subgroups.

In SDMA systems, to serve a large number of devices, a base station has to be equipped with a large number of antennas,

The financial support of InterDigital, UK and that of the ERC's Beam-Me-Up project is gratefully acknowledged. which would result in a significant hardware complexity and power consumption. Thus, to accommodate a large number of devices, while mitigating the hardware complexity, a carefully designed transmit precoder (TPC) can be employed before transmission. In communication systems, the quality-of-service can be defined in terms of the bit error ratio (BER). Hence, designing the TPC based on minimizing the BER of the system should be considered.

A powerful adaptive beamforming scheme was proposed for minimizing the BER of binary phase shift keying (BPSK) constellation in [6], [7], where the beamforming weights of the receiver were designed using the direct minimum bit error ratio (MBER) criterion. Chen et al. extended the MBER beamforming receiver also to SDMA-aided quadrature amplitude modulation in rank-deficient systems in additive white Gaussian noise (AWGN) channels [8]. More recently, the MBER framework was also proposed for a non-regenerative multipleinput multiple-output (MIMO) relay aided system by Dutta et al. [9]. However, these designs determine the beamforming weights at the receiver based on the MBER criterion. More explicitly, these work focus on the design of combining matrix at the receiver. In contrast to the above MBER receivers, Chen et al. also studied MBER aided beamforming at the transmitter [10] using particle-swarm optimization, where the system considered is of full-rank, i.e. the number of data streams is equal to the number of transmit antennas. Furthermore, Pitakdumrongkija et al. designed an MBER based TPC for a system employing maximum-likelihood (ML) detection [11].

Against this background, our contributions are summarized as follows.

1) We accommodate a large number of IoT devices, while mitigating the hardware complexity where the base station serves all devices at a reduced individual data rate, when the number of devices is increased. In contrast to the aforementioned work, in our system, the base station is capable of supporting more downlink signal streams than the number of antennas, using a TPC. Hence, this system is termed as a rank-deficient system [12]. The careful design of the TPC is critical in rank-deficient systems for accommodating a large number of IoT devices. Hence, in order to design the MBER TPC weights that minimize the BER for rank-deficient systems, we invoke the particle swarm optimization (PSO) algorithm [13].

2) We show using simulation results that the proposed MBER TPC is capable of significantly outperforming the LMMSE TPC, which produces an error floor in the rank-deficient systems.

The rest of the paper is organized as follows. Sec. II describes 
the system model considered, while Sec. III presents the MBER problem formulation. Sec. III and Sec. IV discuss the PSO algorithm and our simulation results, while our conclusions are presented in Sec. V.

Notations: We use $\mathbf{A}$ for matrices and $\mathbf{a}$ for vectors, while we use $\mathcal{C N}$, and i.i.d. to denote complex-valued normal distribution, and independent and identical distribution, respectively.

\section{SySTEM MODEL}

Consider a downlink (DL) scenario, where a base station is equipped with $N_{t}$ transmit antennas and communicates with $K$ IoT devices in different angular directions. We presume that all IoT devices are stationary and are equipped with a single antenna, which are mounted on lamp posts, or buildings, etc., so that the channel between the base station (BS) and the IoT device may exhibit line-of-sight (LOS) dominance. Furthermore, in mmWave communications, the channel is predominantly of LOS nature [14], [15]. Hence, we consider a Rician distributed channel between the BS and the IoT devices, which is more practical in the considered scenario. Moreover, when $K \leq N_{t}$, the system is said to be of full-rank, or rank- $K$ system, since the number of IoT devices served is less than the number of transmit antennas at the BS. In this case, the BS can transmit $K$ symbols using $N_{t}$ antennas, since the dimension of the symbol vector is less than or equal to $N_{t}$. On the other hand, when $K>N_{t}$ the system becomes rank-deficient, since the BS is serving more IoT devices than the number of transmit antennas. In other words, the dimension of the symbol vector is greater than $N_{t}$. Let us consider a DL scenario, where the BS transmits $\mathbf{x}=\left[x_{1} \ldots x_{K}\right]^{T}$ to $K$ IoT devices in the same time-frequency radio resource. Before transmission, the signals are precoded using the TPC matrix $\mathbf{P}$ of size $N_{t} \times K$. Then, the signal vector $\mathbf{y}$ received in the downlink is given by

$$
\mathbf{y}=\mathbf{H P x}+\mathbf{n},
$$

where $\mathbf{H}$ has Rician distributed elements, representing both the line-of-sight (LOS) and non-line-of-sight (NLOS) components, which is given by [16]

$$
\mathbf{H}=\sqrt{\frac{L}{L+1}} \mathbf{H}_{\mathrm{LOS}}+\sqrt{\frac{1}{L+1}} \mathbf{H}_{\mathrm{NLOS}},
$$

where we have $\mathbf{H}_{\mathrm{LOS}}=\left[\begin{array}{ll}\mathbf{h}_{1} & \mathbf{h}_{2} \ldots \mathbf{h}_{K}\end{array}\right]$ and $\mathbf{h}_{k}=\left[1 \exp \left(j \frac{2 \pi}{\lambda} d \cos \theta_{k}\right) \ldots \exp \left(j \frac{2 \pi}{\lambda}\left(N_{t}-1\right) d \cos \theta_{k}\right]^{T}\right.$ [15], while $\theta_{k}$ is the angle of departure at the BS to the $k^{\text {th }}$ user, $d$ is the distance between the transmit antennas, $\lambda$ is the wavelength of the signal and $L$ is the Rician fading factor, which represents the channel power ratio between the LOS and NLOS components. Additionally, the entries in $\mathbf{H}_{\mathrm{NLOS}}$ are complex Gaussian variables with distribution $\sim \mathcal{C N}(0,1)$ and uniformly distributed phase. It is instructive to note that the channel matrix $\mathbf{H}$ is uncorrelated in time and it is of size $K \times N_{t}$. Furthermore, $\mathbf{n}=\left[n_{1} \ldots n_{K}\right]^{T}$ is the noise vector of size $K \times 1$ whose entries are i.i.d. with distribution $\mathcal{C N}\left(0, \sigma^{2}\right)$ of mean 0 and of variance $\sigma^{2}$, while $\mathbf{y}=\left[y_{1} \ldots y_{K}\right]^{T}$ is the received vector of size $K \times 1$ and the variance of the channel $\mathbb{E}[\|\mathbf{H}\|]^{2}=K N_{t}$ and $\mathbf{x}=\left[x_{1} \ldots x_{K}\right]^{T}$ is the signal vector of size $K \times 1$, where $x_{k}$ denotes the symbol of the IoT device $k$ drawn from the M-ary QAM set. We note that any complex number can be represented by its real part $(\mathcal{R}\{\}$. plus imaginary part $(\mathcal{I}\{\}$.$) , i.e. \mathcal{R}\{\}+.\mathcal{I}\{$.$\} . Bearing this in$ mind, we find the probability of error for our system.

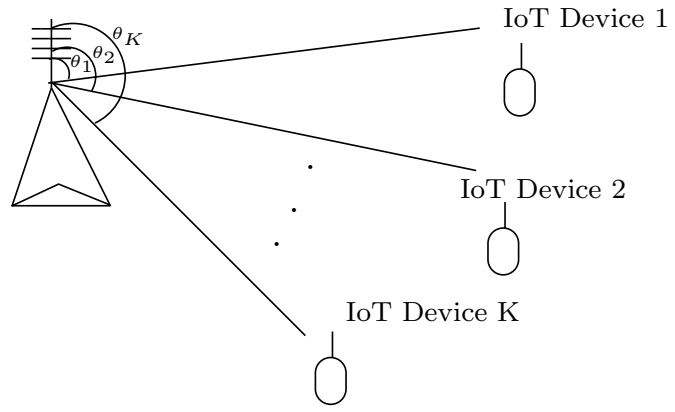

Fig. 1: Base station transmitting in the DL using $N_{t}$ antennas to $K$ IoT devices that are separated in the angular domain.

The proposed TPC relies on the idealized simplifying assumption of having perfect channel state information (CSI) knowledge at the transmitter. However, in practice the CSI can only be obtained over a feedback channel in frequencydivision duplex (FDD) systems, or by exploiting the reciprocity in time-division duplex (TDD) systems [17].

In the next section, we present the MBER problem formulation of designing the TPC matrix P. We employ our MBER solution to rank-deficient systems, where the number of IoT devices is greater than the number of antennas at the BS. We note that the individual device rate may be reduced to serve more devices and vice-versa. However, the sum-rate is constant, since the total rate of the system depends on the number of dimensions available, which is $\min \left\{N_{t}, K\right\}$ [18].

\section{Particle SWARm Optimization Aided MBER PRECODING}

Here we only present the probability of error expression of a 4-QAM constellation, while that of higher-order constellations can be derived by following the approach of [8]. The probability of error for 4-QAM or QPSK modulation can be split into real and imaginary parts, where each part behaves as a BPSK constellation. Thus, the average BER expression for the in-phase (real part) component of the QPSK modulation is given by [19]

$$
\begin{aligned}
& P_{e R}(\mathbf{P})=\frac{1}{K M^{K}} \sum_{q=1}^{M^{K}} \sum_{k=1}^{K} \\
& Q\left(\frac{\operatorname{sgn}\left(\mathcal{R}\left[x_{k}^{(q)}\right]\right) \mathcal{R}\left[\mathbf{h}_{k} \mathbf{P} \mathbf{x}^{(q)}\right]}{\sigma_{n} / \sqrt{2}}\right),
\end{aligned}
$$

where $M$ is the size of the constellation and $K$ is the number of IoT devices. Here we assume that all the devices receive symbols selected from the same constellation. To elaborate further, $\mathbf{x}^{(q)}$ is the $q^{\text {th }}$ transmit signal vector out of $M^{K}$ possible transmit symbol vectors, where $1 \leq q \leq M^{K}$, while $x_{k}^{(q)}$ is the $k^{t h}$ element of $\mathbf{x}_{q}$ and $\sigma_{n} / \sqrt{2}$ is the noise standard deviation per dimension. Similarly, the average BER expression for the quadrature-phase (imaginary part) component of the QPSK modulation is given by [19]

$$
\begin{aligned}
& P_{e I}(\mathbf{P})=\frac{1}{K M^{K}} \sum_{q=1}^{M^{K}} \sum_{k=1}^{K} \\
& Q\left(\frac{\operatorname{sgn}\left(\mathcal{I}\left[x_{k}^{(q)}\right]\right) \mathcal{I}\left[\mathbf{h}_{k} \mathbf{P x}^{(q)}\right]}{\sigma_{n} / \sqrt{2}}\right) .
\end{aligned}
$$


Thus, the total average BER of the QPSK constellation is given by

$$
P_{e}(\mathbf{P})=\frac{P_{e R}(\mathbf{P})+P_{e I}(\mathbf{P})}{2} .
$$

We have to design the TPC $\mathbf{P}$ which minimizes $P_{e}$. Hence, the optimization problem can be formulated as

$$
\begin{aligned}
\mathbf{P}_{\mathrm{MBER}}= & \min P_{e}(\mathbf{P}) \\
& \text { s.t. }\|\mathbf{P x}\|_{\mathrm{F}}^{2} \leq P_{t},
\end{aligned}
$$

where $P_{t}$ is the total transmit power.

The problem in (6) is a non-linear optimization problem and it is typically solved using an iterative gradient based approach, such as sequential quadratic programming (SQP) [20] or by invoking PSO algorithms, as in [10], [21]. However, SQP imposes a high complexity in terms of number of computations as shown in [10]. Therefore, we resort to the PSO algorithm, since it strikes a compelling performance vs complexity compromise. Hence, to solve the optimization problem of (6), we invoke the PSO algorithm. First, we convert the constrained problem in (6) to an unconstrained problem with a penalty function, which is formulated as:

$$
F(\mathbf{P})=P_{e}(\mathbf{P})+G(\mathbf{P}),
$$

where $G(\mathbf{P})$ is the penalty function given by

$$
G(\mathbf{P})=\left\{\begin{array}{lr}
0, & \mathbb{E}\left[\|\mathbf{P} \mathbf{x}\|^{2}\right]-P_{t} \leq 0 \\
\lambda\left(\mathbb{E}\left[\|\mathbf{P x}\|^{2}\right]-P_{t}\right), & \mathbb{E}\left[\|\mathbf{P x}\|^{2}\right]-P_{t}>0
\end{array},\right.
$$

while $\lambda$ is the penalty factor chosen appropriately.

Thus, the objective problem in (6) is now reduced to

$$
\mathbf{P}_{\mathrm{MBER}}=\min _{\mathbf{P} \in \mathcal{S}^{N} \times K} F(\mathbf{P}) .
$$

The basic idea of PSO is that there is a swarm of particles, where each particle searches for an optimum position value in a search space [13]. Furthermore, each particle remembers the best location where it has been throughout the search and updates its position using the appropriate velocity as the particle's position evolves. The optimum solution is given by the specific particle, which has the best position amongst all other particles.

In our problem, the particle $\mathbf{P}_{i}$ evolves in the search space $\mathcal{S}^{N \times K}$, where $\mathcal{S} \in(-1,1)+j(-1,1)$, which forms a square area in the complex plane. In other words, the entries in the TPC matrix associated with the particle $\mathbf{P}_{i}$ are constructed from the search space $\mathcal{S}$. The top-level view of the algorithm is described below and it is also shown in Fig. 2. A more detailed step by step PSO algorithm is presented in [10], [13], [21].

In PSO, the matrix $\mathbf{P}_{1}$ of the first particle in the swarm is initialized to the LMMSE solution, while rest of the particles are initialized randomly in the search space $\mathcal{S}$.

Then, the cost function is computed using (9) plus the individual particles' best location $\mathbf{P b}$, which represents their best local position that minimizes (9), is updated [21]. Furthermore, the group's best location $\mathbf{G b}$, which represents the best position observed for all the particles that minimizes (9), is also updated according to [10], [21]. Given the new locations, now the particles' velocities are updated, which

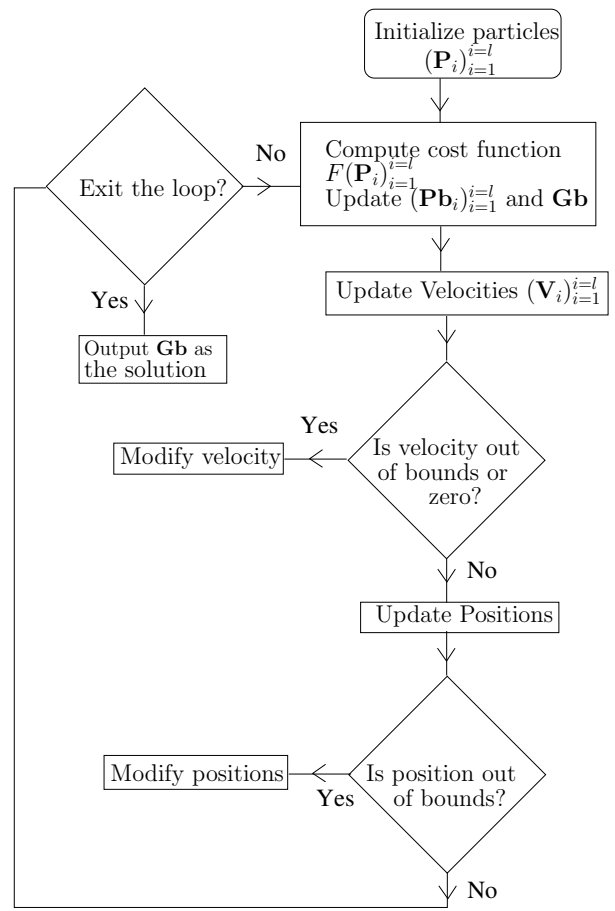

Fig. 2: Flow chart representing the PSO algorithm.

TABLE I: System parameters.

\begin{tabular}{lr}
\hline \hline Parameters & Values \\
\hline Number of Particles & 40 \\
Modulation & QPSK/BPSK \\
$N_{t}$ & 3,4 \\
$K$ & $4,5,7$ \\
$L$ & {$[10,20,30] \mathrm{dB}$} \\
Code rate & $1 / 2$ \\
Constraint length & 7 \\
Generator polynomials (in octal) & 171,133 \\
$\theta$ & Variable \\
\hline
\end{tabular}

are modified if the velocities approach zero [13]. Given the updated velocities, the particles' positions are then updated. This process is repeated until it reaches the maximum number of iterations or convergence of (9). In the next section we demonstrate using our simulation results that for rank-deficient systems the MBER assisted TPC outperforms the LMMSE based system.

\section{Simulation Results}

In this section, we present simulation results for both a rank$K$ system, where $K \leq N_{t}$ and for a rank-deficient system, where we have $K>N_{t}$. In these simulations, the total number of particles used is 40 . To reduce the simulation time, we have considered $N_{t}=3,4$ and $K=4,5,7$ users. However, the proposed algorithm can be readily applied to larger $N_{t}$ and $K$ values. The system parameters used in this paper are summarized in Table I.

Rank-K system: Fig. 3 shows the uncoded BER performance of both the LMMSE aided TPC and the MBER assisted TPC for the rank 4 system, when $N_{t}=4$ and $K=4$.In this configuration, a total of 4 QPSK symbols are transmitted to $K=4$ angularly separated IoT devices using $N_{t}=4$ transmit antennas. In other words, the normalized system load (NSL), which is the ratio of the number of transmitted symbols to 


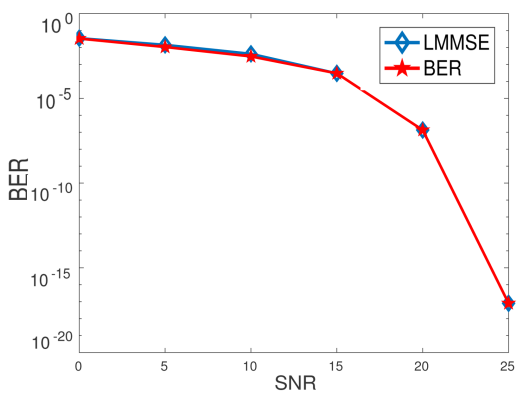

(a)

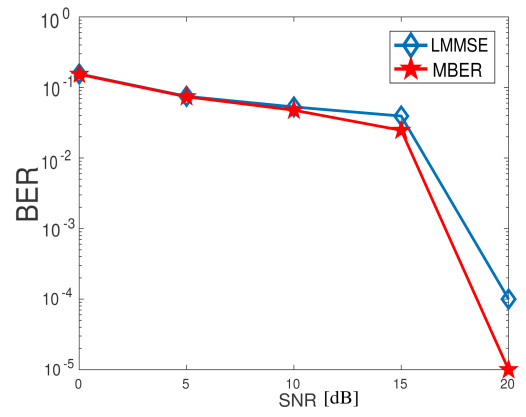

(b)

Fig. 3: BER performance when $N_{t}=4$ and $K=4$, (i.e. $N S L=1$ ) (a) The beam directions are $\theta^{\circ}=\{0,30,-10,-25\}$. (b) The beam directions are $\theta^{\circ}=\{0,10,-10,-25\}$. The simulation parameters are listed in Table I.

the number of transmit antennas, is NSL $=1$. It is shown in Fig. 3 (a) that when the devices are widely separated in the angular domain, i.e. $\theta^{\circ}=\{0,30,-10,-25\}$, the performance of the LMMSE and MBER solutions is similar. This is expected, because in the rank-K systems when devices are widely separated, the LMMSE aided TPC succeeds in efficiently mitigating the inter-device-interference (IDI). However, when the angular spread amongst the devices is low, i.e. $\theta^{\circ}=\{0,10,-10,-25\}$, the LMMSE aided TPC fails to remove the IDI. In contrast to LMMSE, the MBER criterion provides a better BER performance, as shown in Fig. 3 (b).

Rank-deficient system: Fig. 4 shows the uncoded BER performance of both the LMMSE aided TPC and the MBER assisted TPC for the rank-deficient system having $N_{t}=4$ and $K=7^{1}$. In this configuration, a total of 7 BPSK symbols are transmitted to $K=7$ IoT devices using $N_{t}=4$ transmit antennas at the BS. We have set $\mathrm{L}$ to be high $(L \rightarrow \infty)$, because the channel is LOS dominant. It is a rank-deficient system, since the number of symbols transmitted is greater than the number of transmit antennas with $\mathrm{NSL}=1.75$. However, the rate of each user is reduced. In this setting, when the devices are widely separated in the angular domain, i.e. we have $\theta^{\circ}=\{0,10,-10,-25,50,-40,90\}$, the MBER aided TPC outperforms the LMMSE assisted system by a significant margin. Thus, the MBER assisted system efficiently mitigates the IDI, while the LMMSE assisted system results in an error

${ }^{1}$ We have considered $N_{t}=4$ and $K=7$ for brevity, however, the proposed algorithm can be readily applied to larger $N_{t}$ and $K$ values.

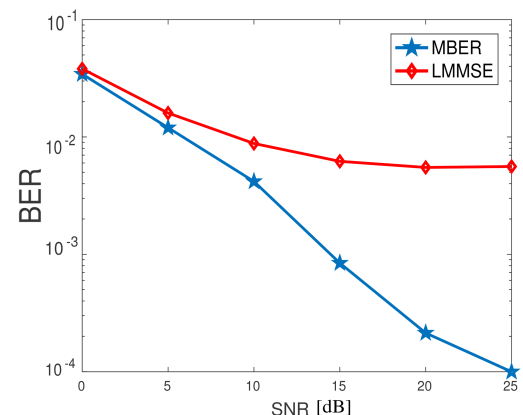

(a)

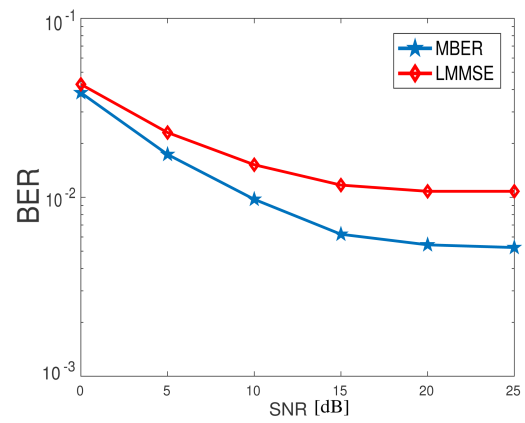

(b)

Fig. 4: BER performance when $N_{t}=4$ and $K=$ 7 (i.e. $N S L=1.75$ ) (a)The beam directions are $\theta^{\circ}=$ $\{0,10,-10,-25,50,-40,90\}$. (b) The beam directions are $\theta^{\circ}=\{0,10,-10,-25,50,-40,60\}$. The simulation parameters are listed in Table I.

floor, as shown in Fig. 4 (a). It is instructive to note that although this is a rank-deficient system, the devices are widely separated in the angular domain. However, when the angular spread between two of the seven devices is too low, i.e. when $\theta^{\circ}=\{0,10,-10,-25,50,-40,60\}$, the MBER aided TPC yields only a limited gain over LMMSE TPC as shown in Fig. 4 (b).

To better understand the behavior of the MBER TPC precoder and also to avoid the error floor observed in Fig. 4 (b), we employ coding to study the performance of both the MBER TPC and the LMMSE aided TPC. Fig. 5 shows the BER of both the convolutional coded and uncoded system for different $L$ values. In this configuration, a total of 5 BPSK symbols are transmitted using $N_{t}=3$ transmit antennas to $K=5$ IoT devices with NSL $=1.66$, hence this is a rank-deficient system. The beam directions of the devices are $\theta^{\circ}=\{0,-20,20,40,-40\}$. It is seen in Fig. 5 that for an uncoded system and large $L(L \rightarrow \infty)$, i.e. when the channel is LOS dominant, the LMMSE TPC yields an error floor, while the MBER aided TPC provides a better BER. However, when the factor $L=30 \mathrm{~dB}$, the MBER TPC provides only a marginal gain for uncoded systems. Moreover, when $L$ is 10 $\mathrm{dB}$, the MBER-assisted system results in an error floor.

On the other hand, for our convolutional coded system using a half-rate code with a constraint length of seven and octally represented generator polynomials of 171 and 133, particularly when the channel is LOS dominant, the BER of the MBER 


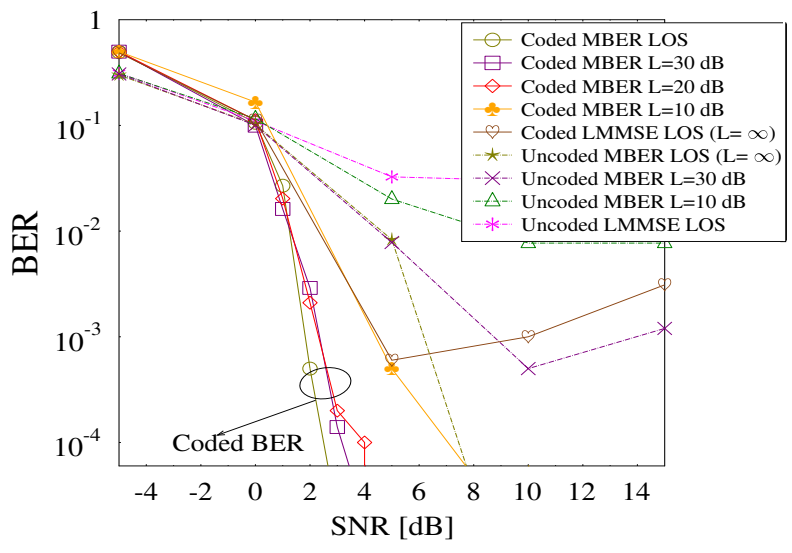

Fig. 5: Coded and uncoded BER of the rank-deficient system with $N_{t}=3$ and $K=5$ (i.e. $N S L=1.66$ ). The beam directions are $\theta^{\circ}=\{0,-20,20,40,-40\}$. The simulation parameters are listed in Table $I$.

based TPC is promising, while the LMMSE based precoder provides only a marginal gain. Furthermore, the BER of the coded MBER system at $L=20 \mathrm{~dB}$ and $30 \mathrm{~dB}$ is similar to that of the system having a perfect LOS.

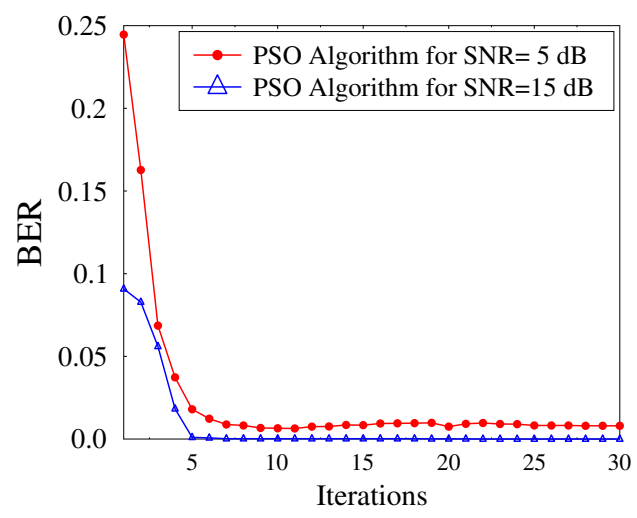

Fig. 6: Convergence performance of PSO aided TPC for the system employing $N_{t}=3$ transmit antennas to support $K=5$ BPSK users with NSL $=1.66$. The beam directions are $\theta^{\circ}=$ $\{0,90,-50,-25,30\}$.

Fig. 6 shows the convergence performance of PSO aided TPC for the system employing $N_{t}=3$ transmit antennas to support $K=5$ BPSK users with $\mathrm{NSL}=1.66$. It is worth observing from Fig. 6 that at SNR of $5 \mathrm{~dB}$ the PSO algorithm converged after 8 iterations, while at SNR of $15 \mathrm{~dB}$ it converged after 5 iterations. The convergence results obtained beyond SNR of $15 \mathrm{~dB}$ are observed to be similar.

\section{CONCLusions}

In this paper, an MBER assisted TPC was proposed for rankdeficient MIMO systems in the context of the IoT. The PSO algorithm was invoked to optimize the non-linearly constrained MBER problem and we demonstrated by our simulation results that the PSO assisted MBER TPC achieves a better BER performance in rank-deficient systems than conventional TPC designs, such as zero forcing and LMMSE aided TPCs, which produce an error floor.

\section{REFERENCES}

[1] P. Ramezani and A. Jamalipour, "Toward the evolution of wireless powered communication networks for the future internet of things," IEEE Network, vol. PP, no. 99, pp. 12-19, 2017.

[2] A. Zanella et al., "Internet of things for smart cities," IEEE Internet of Things Journal, vol. 1, no. 1, pp. 22-32, Feb 2014.

[3] L. Song, S. Wu, and H. Wang, "SIMPLEX: Symbol-level information multiplex," IEEE Internet of Things Journal, vol. 3, no. 5, pp. 757-766, Oct 2016.

[4] Z. Ding, L. Dai, and H. V. Poor, "MIMO-NOMA design for small packet transmission in the internet of things," IEEE Access, vol. 4, pp. 1393-1405, 2016.

[5] R. Tian et al., "Overlapping user grouping in IoT oriented massive MIMO systems," IEEE Access, vol. PP, no. 99, pp. 1-1, 2017.

[6] S. Chen, L. Hanzo, and N. N. Ahmad, "Adaptive minimum bit error rate beamforming assisted receiver for wireless communications," in Proc. ICASSP, vol. 4, April 2003, pp. IV-640-3 vol.4.

[7] L. Fan, H. Zhang, and C. He, "Minimum bit error rate beamforming for pre-FFT OFDM adaptive antenna array," in Proc. VTC (Fall), vol. 1, Sept 2005, pp. 359-363.

[8] S. Chen, J. Zhang, X. Mu, and L. Hanzo, "Minimum bit error rate beamforming receiver for space-division multiple-access based quadrature amplitude modulation systems," in Proc. WCNC, April 2012, pp. 498-502.

[9] A. K. Dutta, K. V. S. Hari, and L. Hanzo, "Linear transceiver design for an amplify-and-forward relay based on the MBER criterion," IEEE Trans. Commun., vol. 62, no. 11, pp. 3765-3777, Nov 2014.

[10] W. Yao, S. Chen, S. Tan, and L. Hanzo, "Minimum bit error rate multiuser transmission designs using particle swarm optimisation," IEEE Trans. Wireless Commun., vol. 8, no. 10, pp. 5012-5017, October 2009.

[11] B. Pitakdumrongkija et al., "Linear precoding with minimum BER criterion for MIMO-OFDM systems employing ML detection," in Proc. ICC, June 2007, pp. 2522-2527.

[12] A. Wolfgang, J. Akhtman, S. Chen, and L. Hanzo, "Iterative MIMO detection for rank-deficient systems," IEEE Trans. Signal Process., vol. 13, no. 11, pp. 699-702, Nov 2006.

[13] A. Ratnaweera, S. K. Halgamuge, and H. C. Watson, "Selforganizing hierarchical particle swarm optimizer with timevarying acceleration coefficients," IEEE Trans. Evolu. Comput., vol. 8, no. 3, pp. 240-255, June 2004.

[14] I. Hemadeh, K. Satyanarayana, M. El-Hajjar, and L. Hanzo, "Millimeter-wave communications: Physical channel models, design considerations, antenna constructions and link-budget," IEEE Commun. Surveys Tuts., vol. PP, no. 99, pp. 1-1, 2018.

[15] K. Satyanarayana, M. El-Hajjar, P. H. Kuo, A. Mourad, and L. Hanzo, "Dual-function hybrid beamforming and transmit diversity aided millimeter wave architecture," IEEE Trans. Veh. Technol., vol. 67, no. 3, pp. 2798-2803, March 2018.

[16] F. Bohagen, P. Orten, and G. E. Oien, "Design of optimal high-rank line-of-sight MIMO channels," IEEE Trans. Wireless Commun., vol. 6, no. 4, pp. 1420-1425, April 2007.

[17] J. Jose et al., "Pilot contamination and precoding in multi-cell TDD systems," IEEE Trans. Wireless Commun., vol. 10, no. 8, pp. 2640-2651, August 2011.

[18] E. Telatar, "Capacity of multi-antenna Gaussian channels," Jun 1995, AT\&T-Bell Laboratories Internal Tech. Memo.

[19] S. Chen, A. Livingstone, and L. Hanzo, "Minimum bit-error rate design for space-time equalization-based multiuser detection," IEEE Trans. Wireless Commun., vol. 54, no. 5, pp. 824-832, May 2006.

[20] J. Nocedal and S. J. Wright, Numerical Optimization, 5th ed. Springer, 1999.

[21] K. K. Soo et al., "Particle-swarm-optimization-based multiuser detector for CDMA communications," IEEE Trans. Veh. Technol., vol. 56, no. 5, pp. 3006-3013, Sept 2007. 